Reference [2] estimates that the penalty for adding a 125 $\mathrm{Mb} / \mathrm{s}$ ASK modulated signal to a $2.488 \mathrm{~Gb} / \mathrm{s}$ PSK signal is about $3.5 \mathrm{~dB}$, and the penalty for adding a $125 \mathrm{Mb} / \mathrm{s}$ subcarrier to a $2.488 \mathrm{~Gb} / \mathrm{s}$ intensity modulated signal is about $1.8 \mathrm{~dB}$. These additional penalties would result in receiver sensitivities of 188 photons/bit for the preamplified subcarrier multiplexed scheme and only 110 photons/bit for the PSK/ASK scheme.

While the sensitivity of our experimental transceiver is a modest $-25 \mathrm{dBm}$, the power budget we achieved is still large enough for a 100 node passive star network with a diameter of $5 \mathrm{~km}$ and a power margin of $6 \mathrm{~dB}$. The PSK/ASK transceiver described in this work will be used in WDM network experiments currently underway at Stanford University.

\section{ACKNOWLEDGMENT}

The authors would like to thank D. Atlas of Lasertron for helpful discussions of PSK receiver design; T. Oslon and Frank Adams of Lightwave Electronics for helpful discussions of Lightwave Electronics' Nd:YAG lasers; and A. Mahapatra of Ramar Corporation for his help with the modulator design.

\section{REFERENCES}

[1] P. Poggiolini and L. G. Kazovsky, "STARNET: an integrated services broadband optical network with physical star topology," in Proc. SPIE 1579, Adianced Fiber Commun. Technol, Boston, MA, 1991, pp. 14-29.
[2] L. G. Kazovsky and P. Poggiolini, "STARNET: a multi-Gbit/s optical LAN utilizing a passive WDM star," J. Lightwave Technol., May 1993.

[3] M. S. Goodman, H. Kobrinski, M. P. Vecchi, R. M. Bulley, and J. L. Gimlett, "The LAMBDANET multiwavelength network: architecture, applications, and demonstrations," IEEE J. Select. Areas Commun., vol. 8, no. 6, pp. 995-1004, Aug. 1990.

[4] B. S. Glance and O. Scaramucci, "Computer-controlled multichannel heterodyne optical communication system," IEEE Photon. Technol. Lett., vol. 3, pp. 83-85, Jan. 1991.

[5] N. R. Dono, P. E. Green, K. Liu, R. Ramaswami, and F. Tong, "A wavelength division multiple access network for computer communication," IEEE J. Select. Areas Commun., vol. 8, no. 6, pp. 983-993, Aug. 1990.

[6] R. Gidron, "TeraNet: a multi-gigabits per second ATM network," Computer Commun., vol. 15, no. 3, pp. 143-152, Apr. 1992.

[7] R. Noé, H. J. Rodler, A. Ebberg, G. Gaukel, B. Noll, J. Wittmann, and F. Auracher, "Comparison of polarization handling methods in coherent optical systems," J. Lightwave Technol, vol. 9, pp. 1353-1366, Oct. 1991

[8] D. A. Atlas and L. G. Kazovsky, "Optical PSK synchronous heterodyne experiments at $560 \mathrm{Mbit} / \mathrm{s}$ through $4 \mathrm{Gbit} / \mathrm{s}$," J. Opt. Commun., vol. 12, pp. 130-137, Dec. 1991.

[9] ANSI X3T9.5 FDDI specification, Rev. 5.1, Sept. 1989.

[10] M. J. Hickey and L. G. Kazovsky, "Combined ASK/PSK modulation format for the STARNET wavelength-division-multiplexed local area network," in Proc. SPIE 1787, Multigigabit Fiber Commun., Boston, MA, 1992.

[11] L. G. Kazovsky and O. K. Tonguz, "ASK and FSK coherent lightwave systems: A simplified approximate analysis," J. Lightwave Technol, vol. 8, pp. 338-352, Mar. 1990.

[12] Y. K. Park, S. W. Granlund, T. W. Cline, L. D. Tzeng, J. S. French, J.-M. P. Delavaux, R. E. Tench, S. K. Korotky, J. J. Veselka, and D. J. DiGiovanni, " $2.488 \mathrm{~Gb} / \mathrm{s}-318 \mathrm{~km}$ repeaterless transmission using erbium-doped fiber amplifiers in a direct-detection system," IEEE Photon. Technol. Lett., vol. 4, pp. 179-182, Feb. 1991.

\title{
The Influence of the Unlocked Characteristics of a Self-Pulsating Laser Diode on Synchronization
}

\author{
Gerald Farrell, Paul Phelan, and John Hegarty
}

\begin{abstract}
All-optical synchronization and timing extraction based on self-pulsating twin section lasers bas been demonstrated. We investigate the variation of the unlocked full-widthat-half-maximum of the fundamental of the RF spectrum of a self-pulsating laser diode. We show for the first time that the value of the unlocked FWHM has a direct influence on the power required to maintain synchronization. We also show that the variation of the FWHM with frequency is dependent on the slope of the absorber bias versus self-pulsation frequency characteristic. The results have important implications for the de-
\end{abstract}

Manuscript received December 1, 1992.

The authors are with Optronics Ireland Research Laboratories, Trinity College, Dublin 2, Ireland

IEEE Log Number 9208629. sign and selection of self-pulsating laser diodes for synchronization applications.

\section{INTRODUCTION}

$T$ HE development of very high capacity transmission and switching systems for future communications networks is being actively pursued. To overcome the speed and architectural limitations of electronics the demand for all-optical functional devices is increasing. One important function in communications switching and transmission systems is synchronization. 
Synchronization using a self-pulsating twin section laser diode is an all-optical function which has been demonstrated in a number of applications. For example, all-optical timing extraction from a data signal has been demonstrated [1], [2]. Recently, we have reported all-optical frequency division [3], [4] and multiplication [5], using a self-pulsating laser diode.

While the underlying principles of self-pulsation have been explored in detail by a number of authors, for example [6], the specific requirements for self-pulsating devices used for synchronization have not been examined in depth. Presently, the only conditions for effective synchronization are that the self-pulsating laser operates at the source wavelength in use and at the appropriate self-pulsating frequency. If these devices are to be used in actual systems, however, there is a need to explore the nature of synchronization in greater detail and establish what factors influence synchronization.

In this letter we show for the first time that the fullwidth-at-half maximum (FWHM) of the fundamental of the unlocked self-pulsating RF spectrum has a direct effect on the optical input power needed to maintain synchronization. We demonstrate that the FWHM varies with the self-pulsation frequency which is controlled by the applied bias to the laser sections. Furthermore, we show that increases in the value of the FWHM correspond to a steeper slope on the self-pulsation frequency versus absorber bias characteristic.

\section{EXPERIMENTAL SETUP}

The experimental setup is shown in Fig. 1. The selfpulsating laser used is a two-section InGaAsP BH device with a length of $500 \mu \mathrm{m}$ and a $4: 1$ gain to absorber section length ratio. This device exhibits strong selfpulsation when the absorber is operated within the selfpulsation regime reported recently [7]. Two such devices were investigated, yielding similar results.

The source laser is a mode locked InGaAsP BH laser operating in a tunable grating external-cavity. Light from this grating external-cavity laser is coupled to the selfpulsating laser using an $8 \mu \mathrm{m}$ (radius) lens-ended fiber. Polarization control and optical isolation are used. The output wavelength of the grating external-cavity laser is adjusted so that it coincides with one of the longitudinal modes of the self-pulsating laser diode. Both lasers are temperature controlled to within $\pm 0.1{ }^{\circ} \mathrm{C}$. An avalanche photodiode with a bandwidth greater than $1.8 \mathrm{GHz}$ is used to observe laser outputs on a spectrum analyser and oscilloscope.

\section{VARIATION IN THE UnLOCKED SElF-PUlSATION FWHM}

The FWHM of the fundamental component of the RF spectrum of the unlocked self-pulsation was measured as a function of the absorber voltage and current, using a spectrum analyser. At each value of absorber voltage and current the self-pulsation frequency was also noted. The gain section current was fixed at $83 \mathrm{~mA}$. It is found that

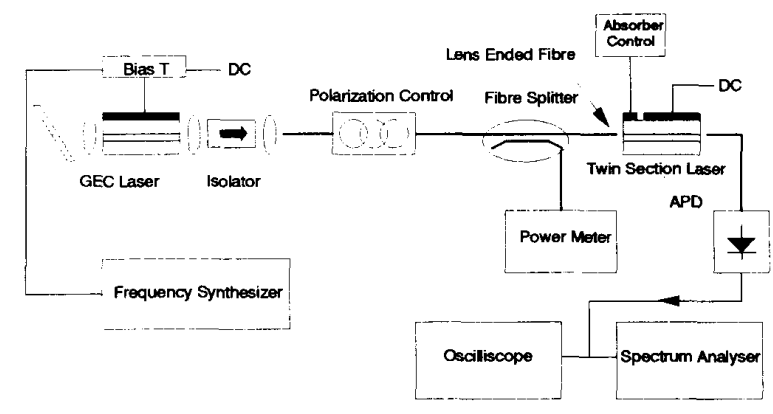

Fig. 1. Experimental setup.

the FWHM varies widely over the range of self-pulsating frequencies. The variation of the FWHM as a function of the self-pulsation frequency is shown in Fig. 2. The minimum FWHM is $273 \mathrm{KHz}$ at $440 \mathrm{MHz}$ and the maximum FWHM is $11.1 \mathrm{MHz}$ at $583 \mathrm{MHz}$. The ratio of the maximum to minimum value of the FWHM is in excess of 40:1.

\section{EFFECT OF THE UNLOCKED FWHM ON SYNCHRONIZATION}

In order to examine how the magnitude of the unlocked FWHM affects synchronization the grating external cavity laser was used to provide a mode-locked input signal to synchronise the self-pulsating laser diode. The pulse duration, observed on a $20 \mathrm{GHz}$ p-i-n photodiode, was maintained at less than 75 ps. The mode-locked laser output frequency was set at a number of values in turn and at each mode-locked frequency synchronization between the mode-locked input signal and the self-pulsating laser diode was achieved. At each frequency used the absorber voltage was adjusted to achieve the lowest noise floor level for the fundamental component of the synchronized selfpulsation signal. At all of the frequencies used the gain section current of the self-pulsating laser diode was fixed at $83 \mathrm{~mA}$ and the operating wavelength of the mode-locked laser was adjusted to match the same longitudinal mode of the self-pulsating laser diode.

The synchronized mode-locked and self-pulsation signals were monitored on a twin-channel oscilloscope triggered on the mode-locked input. The mode-locked input optical power was then reduced to a level at which synchronization was just lost. Fig. 3 shows the variation in the value of the input optical power needed to just maintain synchronization as a function of the synchronization frequency. The variation of the FWHM is also plotted in Fig. 3 for comparison. For low values of FWHM the input optical power required is about $0.6 \mu \mathrm{W}$ (at $440 \mathrm{MHz}$ ), while at frequencies at which the unlocked FWHM is higher the input power required is larger. For example, at $583 \mathrm{MHz}$ where the FWHM is greater than $10 \mathrm{MHz}$, the input optical power needed is $2.14 \mu \mathrm{W}$. Operating at a frequency at which the FWHM is close to maximum will therefore involve a power penalty since an extra $5.5 \mathrm{~dB}$ of optical power is needed to maintain synchronization. Such 


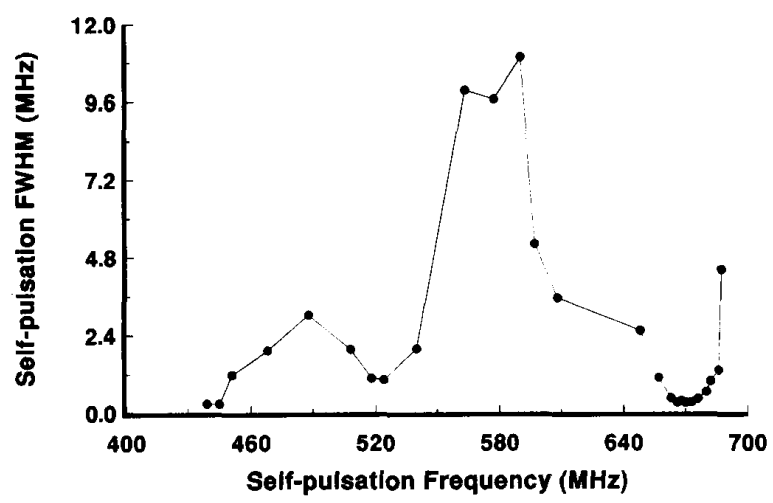

Fig. 2. The variation of the FWHM with the unlocked self-pulsation frequency.

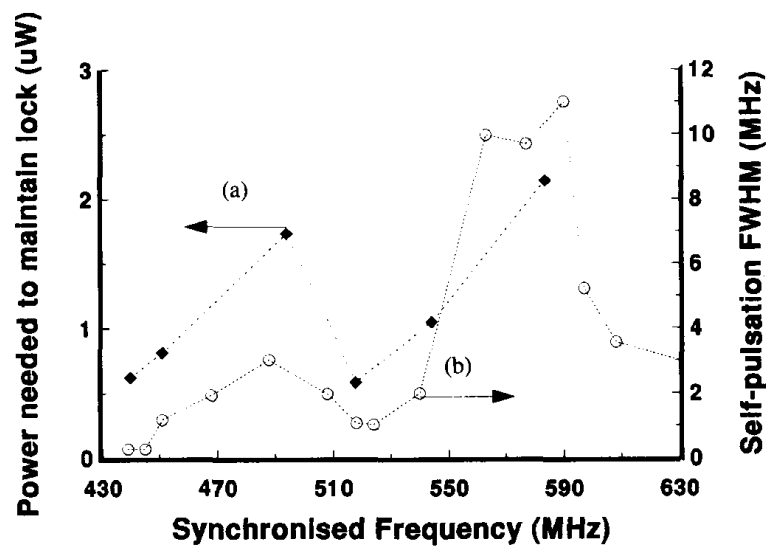

Fig. 3. Plot (a) shows the input optical power needed to maintain synchronization versus the synchronization frequency. The variation of FWHM with frequency is also shown in plot (b) for comparison.

a power penalty would be very significant for a transmission system in which the operating span is attenuation limited.

We have previously shown that, near the synchronized frequency, the value of the noise floor relative to the level of the synchronized fundamental component varies with the input optical power [4]. Above a certain input optical power level saturation occurs and ratio of the synchronized component to the noise floor does not improve, while below this power level the noise floor level increases as the input optical power is decreased.

For the same input optical power the relative noise floor was measured at two operating frequencies, one of which had a low unlocked FWHM value $(440 \mathrm{MHz})$ and the other which had a high FWHM value $(583 \mathrm{MHz})$. For an input optical power of $3 \mu \mathrm{W}$ the noise floor is $42 \mathrm{~dB}$ below the synchronized peak level at $440 \mathrm{MHz}$, while at $583 \mathrm{MHz}$ the noise floor is $31 \mathrm{~dB}$ below the synchronized peak level. This demonstrates that if the optical power available for synchronization is limited, the quality of synchronization will suffer if the self-pulsating laser is

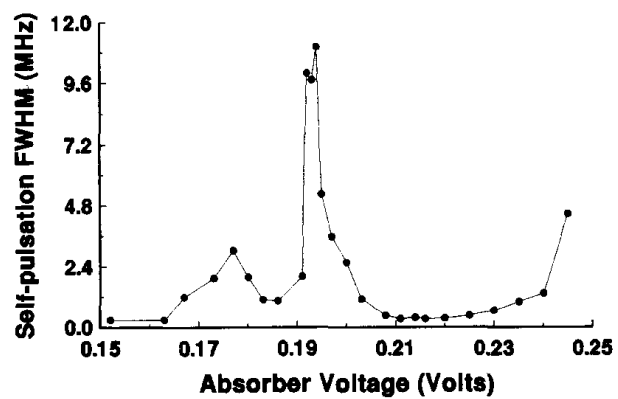

(a)

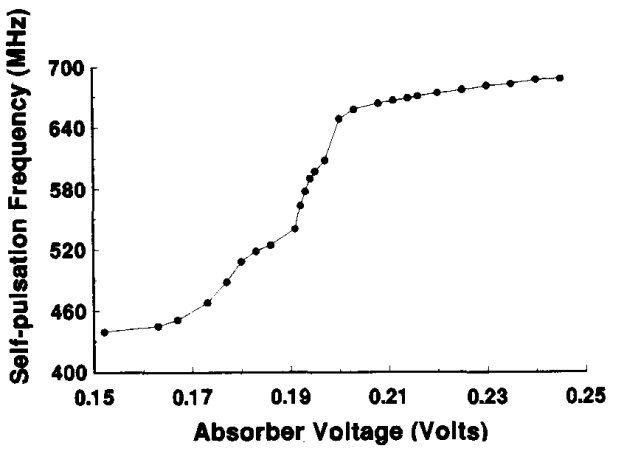

(b)

Fig. 4. The effect of the absorber voltage on the device characteristics at a constant gain section current. (a) The FWHM as a function of absorber voltage and (b) the unlocked self-pulsation frequency versus absorber voltage.

operated at a frequency at which the unlocked FWHM is large.

\section{DeVICE Characteristics AND THE FWHM}

As indicated above for a gain section current of $83 \mathrm{~mA}$ the FWHM and self-pulsation frequency were measured as a function of the absorber bias. Fig. 4(a) and (b) show how the FWHM and the self-pulsation frequency vary with absorber voltage. It is found that as the self-pulsation frequency increases with absorber voltage the rate of change of frequency with absorber voltage varies. The value of the FWHM follows variations in rate of change of frequency with voltage. The lowest FWHM occurs at $440 \mathrm{MHz}$ where the rate of change of frequency with absorber voltage is $0.451 \mathrm{MHz} / \mathrm{mV}$. The highest FWHM occurs at $583 \mathrm{MHz}$ where the rate of change of 13 $\mathrm{MHz} / \mathrm{mV}$. The ratio of the maximum to minimum rate of change is $29: 1$, which is similar to the ratio of the maximum to minimum FWHM.

The variation of self-pulsation frequency with absorber current was also measured and again it was found that the self-pulsation FWHM varied with the rate of change of self-pulsation frequency with absorber current.

These results are significant in two ways. Firstly, the results indicate that in the design of new twin section self-pulsating laser diodes for synchronization applications it is desirable to keep the slope of the self-pulsation frequency versus absorber bias characteristic as low as 
possible. Secondly, when selecting existing self-pulsating lasers the self-pulsation frequency versus absorber bias characteristic can be used to easily quantify the best operating frequencies for the device.

\section{CONCLUSION}

We have demonstrated that the value of the unlocked FWHM of a self-pulsating laser diode has a significant effect on the input power needed for synchronization. Therefore, in developing new twin-section lasers for alloptical synchronization we conclude that it is not enough to ensure that the appropriate self-pulsation frequency can be attained to match the input operating frequency. It is important to ensure that the FWHM of the unlocked self-pulsation is low at the operating frequency in use to avoid a power penalty.

We have also shown that a low FWHM value at a particular frequency is associated with a low rate of change of self-pulsation frequency with absorber bias. This is significant for the design of new twin section self-pulsating laser diodes and for the selection of operating points for existing devices.

\section{ACKNOWLEDGMENT}

The authors would like to thank M. J. Robertson of British Telecom Laboratories for providing the lasers used in this work.

\section{REFERENCES}

[1] M. Jinno and T. Matsumoto, "Optical retiming regenerator using $1.5 \mu \mathrm{m}$ multi-electrode DFB LDs," Electron. Lett., vol. 25 , no. 20 , pp. 1332-1333, 1989.

[2] P. E. Barnsley, G. E. Wickens, H. J. Wickes, and D. M. Spirit, “Al optical clock recovery at $5 \mathrm{Gbit} / \mathrm{s}$ using a two contact semiconductor self-pulsating laser amplifier," OSA Opt. Amp. App., Snowmass, CO, July 1991.

[3] G. Farrell, P. Phelan, and J. Hegarty, "All-optical synchronisation with frequency division using a self pulsating laser diode," Electron. Lett., vol. 28, no. 8, pp. 738-739, 1992.

[4] P. Phelan, G. Farrell, and J. Hegarty, "All-optical synchronisation and frequency division of mode-locked pulses," IEEE Photon. Technol. Lett., vol. 4, pp. 930-932, Aug. 1992.

[5] —, "All-optical synchronisation and multiplication of the frequency of modelocked signals," IEEE Photon. Technol. Lett., Dec. 1992 .

[6] M. Ueno and R. Lang, "Conditions for self-sustained pulsation and bistability in semiconductor lasers," J. Appl. Phys., vol. 58, no. 4, pp. 1689-1692, 1985.

[7] G. Farrell, P. Phelan, and J. Hegarty, "Self pulsation operating regime for the Absorber of a twin section laser diode," Electron. Lett., vol. 27, no. 16, pp. 1403-1404, 1991.

\title{
Millimeter Wave, Multigigahertz Optical Modulation by Feedforward Phase Noise Compensation of a Beat Note Generated by Photomixing of Two Laser Diodes
}

\author{
Olav Solgaard, John Park, John B. Georges, Petar K. Pepeljugoski, and Kam Y. Lau
}

\begin{abstract}
In this letter, we describe a technique for high frequency (up to $100 \mathrm{GHz}$ ), narrow-band $(\sim \mathrm{GHz}$ ) optical modulation by encoding the millimeter wave signal onto a beat note, produced by photomixing of two $1.3 \mu \mathrm{m}$ DFB lasers. The phase noise on the beat note is compensated by a feedforward technique, using a low frequency external optical modulator, which simutaneously encodes the information to be transmitted. The modulation band can be tuned by varying the lasing frequency of one or both of the lasers. We investigated the fundamental performance limit of this technique and demonstrated transmission of a pulsed RF signal at $\mathbf{4 0} \mathbf{G H z}$
\end{abstract}

Manuscript received February 1, 1993. This work was supported by DARPA and Rome Laboratory.

The authors are with the Department of Electrical Engineering and Computer Science, University of California at Berkeley, Berkeley, CA 94720 .

IEEE Log Number 9208622.
7 HE development of optical modulation techniques at frequencies of tens of gigahertz is motivated to a large part by potential applications in microwave and millimeter-wave transmission in optical fiber, such as phased array antenna systems. Most of these systems operate over a relatively narrow band (a few gigahertz), although the center frequency may vary over a wide range (as in frequency hopping schemes). Recent work in highfrequency, narrow-band modulation include direct modulation at the inter modal frequency of laser diodes [1], [2], and traveling wave external modulators. One drawback to these schemes is that, once implemented, the operational frequency band remains fixed.

This paper reports a technique for narrow-band (a few gigahertz) millimeter-wave optical modulation where the 